•研究报告・

\title{
喜旱莲子草对模拟全天增温的可塑性：引入地和原 产地种群的比较
}

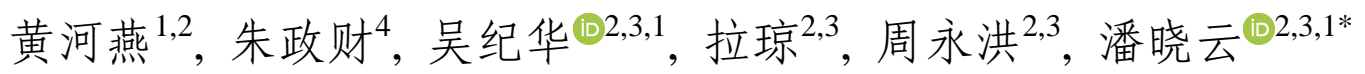

1. 复旦大学生物多样性与生态工程教育部重点实验室, 上海 200438; 2. 西藏大学-复旦大学生物多样性与全球变化联合实验室, 拉萨 850000; 3. 西藏大学生命科学系与生物多样性研究所, 拉萨 850000; 4. 广州市增城区林业和园林科学研究所, 广州 511300

摘要: 植物可以通过关键功能性状的表型可塑性来适应气候变暖背景下环境温度的增加。表型可塑性增强进化假说(evolution of increased phenotypic plasticity hypothesis)认为外来植物在引入地进化出了更强的表型可塑性。以往对该假说的验证多集中 于外来植物对光照、水分、养分、邻体以及天敌等的可塑性进化, 而对增温条件下植物生长和功能性状可塑性进化的研究相 对较少。仅有的几项研究多集中在温带地区，且多集中于研究植物生长相关的性状，而对植物的抗性和草食作用对增温的响 应的关注相对较少。本研究采用同质园实验比较了喜早莲子草(Alternanthera philoxeroides)引入地(中国)和原产地(阿根廷)各8 个种群的生物量、功能性状和草食作用在热带地区(广州市增城区)对模拟全天增温 $2^{\circ} \mathrm{C}$ 的响应差异。结果表明: (1)模拟全天增 温显著降低了喜旱莲子草总生物量(-7.8\%)、陉藏根生物量(-12.8\%)、分枝强度(-11.6\%)和茎端取食率(-34.4\%)。(2)模拟全天 增温造成的引入地种群总生物量降低幅度大于原产地种群; 模拟全天增温使引入地种群的比茎长和茎端取食率降低, 而原产 地种群则相反。(3)无论是否模拟全天增温，引入地种群的咜藏根生物量 $(+31.5 \%) 、$ 分枝强度 $(+38.5 \%)$ 、比茎长 $(+30.2 \%) 、$ 根 冠比(+24.5\%)和比叶面积(+20.0\%)均高于原产地种群, 茎端取食率则低于原产地种群(-35.8\%)。这些结果表明, 热带地区全天 增温 $2^{\circ} \mathrm{C}$ 对喜旱莲子草是一种胁迫; 引入地种群的生物量对模拟全天增温 $2^{\circ} \mathrm{C}$ 的响应更强, 而其株形相关性状(比茎长)和草食 作用(茎端取食率)对模拟全天增温的可塑性方向与原产地种群相反。由于引入地种群在热带地区模拟全天增温条件下生物量 的下降和草食作用的增加明显高于原产地种群, 因此在未来全球气候变暖的背景下, 热带地区温度升高可能不利于喜旱莲子 草种群多度的增加。

关键词: 增温; 表型可塑性; 植物入侵; 进化

黄河燕, 朱政财, 吴纪华, 拉琼, 周永洪, 潘晓云 (2021) 喜早莲子草对模拟全天增温的可塑性: 引入地和原产地种群的比较. 生物多样性, 29, 419-427. doi: 10.17520/biods.2020387.

Huang HY, Zhu ZC, Wu JH, La Q, Zhou YH, Pan XY (2021) Phenotypic plasticity of Alternanthera philoxeroides in response to simulated daily warming: Introduced vs. native populations. Biodiversity Science, 29, 419-427. doi: 10.17520/biods.2020387.

\section{Phenotypic plasticity of Alternanthera philoxeroides in response to simulated daily warming: Introduced vs. native populations}

Heyan Huang ${ }^{1,2}$, Zhengcai Zhu ${ }^{4}$, Jihua Wu ${ }^{(2,3,1}$, Qiong La ${ }^{2,3}$, Yonghong Zhou ${ }^{2,3}$, Xiaoyun Pan ${ }^{\left(1,3,1^{*}\right.}$

1 Ministry of Education Key Laboratory for Biodiversity Science and Ecological Engineering, Fudan University, Shanghai 200438

2 Tibet University-Fudan University Joint Laboratory for Biodiversity and Global Change, Lhasa 850000

3 Institute of Biodiversity, Department of Biology, Tibet University, Lhasa 850000

4 Guangzhou Zengcheng Institute of Forestry and Landscape Architecture, Guangzhou 511300

\section{ABSTRACT}

Aims: The evolution of increased phenotypic plasticity hypothesis predicts that populations in the introduced range of an invasive species have evolved greater plasticity than populations in the native range. Studies of this hypothesis mostly focused on the plastic evolution of invasive plants to light, water, nutrients, neighboring plants, and natural enemies. However, there are relatively few studies focusing on the evolution of plasticity in plant growth and functional

收稿日期: 2020-10-08; 接受日期: 2021-01-09

基金项目: 国家自然科学基金(41771053; 32071659; 32030067)

* 通讯作者 Author for correspondence. E-mail: xypan@fudan.edu.cn 
traits in response to warming. The few existing studies are concentrated in temperate regions and focus on growth-related traits, but relatively little attention has been paid to tropical regions and herbivory-related traits. To address this gap, we conducted an experiment with Alternanthera philoxeroides to study whether introduced and native populations of invasive plants differ in biomass, important functional traits and herbivory resistance in response to daily warming. Specifically, we addressed the following questions: (1) How do biomass, functional traits and herbivory damage of A. philoxeroides respond to simulated daily warming? (2) Do these responses differ between introduced and native populations of $A$. philoxeroides?

Methods: We conducted a field experiment in Zengcheng District (113.87 E, 23.33 $\left.{ }^{\circ} \mathrm{N}\right)$, Guangzhou City, Guangdong Province in which we grew eight populations of the invasive plant $A$. philoxeroides collected from both the introduced range (China) and the native range (Argentina) under ambient temperature and a condition of simulated daily warming of $2^{\circ} \mathrm{C}$. After eight weeks of growth, we harvested all plants and measured the following variables: (1) biomass (i.e. total biomass and storage root biomass), (2) functional traits (i.e. branching intensity, specific stem length, root-to-shoot ratio and specific leaf area, and (3) herbivory damage (i.e. relative feeding area and stem-tip feeding proportion).

Results: Simulated daily warming of A. philoxeroides significantly reduced total biomass (-7.8\%), storage root biomass $(-12.8 \%)$, branching intensity $(-11.6 \%)$ and stem-tip feeding proportion $(-34.4 \%)$. The reduction in total biomass caused by the daily warming was greater in the introduced than in the native populations. Simulated daily warming reduced specific stem length and stem-tip feeding proportion of the introduced populations, while the native populations showed the opposite pattern. Regardless of simulated daily warming or not, storage root biomass $(+31.5 \%)$, branch strength (+38.5\%), specific stem length $(+30.2 \%)$, root-to-shoot ratio $(+24.5 \%)$ and specific leaf area $(+20.0 \%)$ of the introduced populations were higher than those of the native populations, although stem-tip feeding proportion was lower $(-35.8 \%)$.

Conclusion: These results indicate that simulated daily warming of $2^{\circ} \mathrm{C}$ in tropical regions is a stressor for the invasive plant A. philoxeroides. Biomass of the introduced populations has stronger plasticity in response to simulated daily warming of ${ }^{2} \mathrm{C}$ than that of the native populations. In response to simulated daily warm ing, plasticity of plant shape-related traits (specific stem length) and herbivory-related traits (stem-tip feeding proportion) of the introduced populations shows the opposite direction to that of the native populations. Given that the reduction in biomass reduction and increase in herbivory increase were greater in the introduced than the native populations, future temperature increases due to global climate change may not be beneficial to the abundance of invasive plant $A$. philoxeroides in the tropics.

Key words: warming; phenotypic plasticity; plant invasion; evolution

\section{气候变暖和生物入侵是影响全球生物多样性} 的重要因素(Nicotra et al, 2010)。气候变暖可通过为 植物提供 “热岛”和影响物种的传播方式等 (McKinney, 2006), 在提高种群多度和扩大分布面 积方面起到重要作用(Buckland et al, 2001)。这种效 应在较高纬度和较高海拔的地区尤为明显(Nicotra et al, 2010)。而与此同时, 越来越多的研究表明, 植 物可以通过关键功能性状的表型可塑性来适应气 候变暖背景下环境温度的增加(Nicotra et al, 2010)。

表型可塑性是指某一基因型在不同环境条件 下产生不同生理或形态表型的特性(Daehler, 2003)。 研究表明, 较高的表型可塑性可以提高物种的生态 位宽度, 使其在更广泛的环境中表达有利的表型 (Richards et al, 2006)。表型可塑性在入侵中可能发 挥重要作用: 一方面, 外来入侵物种可能比非入侵 种或本地物种具有更高的表型可塑性(Richards et al, 2006); 另一方面, 表型可塑性增强进化假说 (evolution of increased phenotypic plasticity hypothesis)推测, 外来植物引入地种群可能比原产 地种群进化出了更强的表型可塑性(Richards et al, 2006)。以往对该假说的验证多集中于外来植物对光 照(张紫妍等, 2015)、水分(Geng et al, 2006)、养分(周 方等, 2017)、邻体(Zhang et al, 2019; 于良瑞等, 2020)以及天敌(Liu et al, 2020)等的可塑性进化, 而 对模拟全天增温条件下入侵植物重要功能性状表 型可塑性进化的研究相对较少(Hyldgaard \& Brix, 2012)。仅有的几项研究多集中在温带(Peng et al, 2019; Zhou \& He, 2020), 并且多集中于关注植物生 长的相关性状(He et al, 2012; Griffith et al, 2014), 而在热带地区开展以及关注植物草食作用及其抗 性的研究相对较少(Paudel et al, 2020)。

喜旱莲子草(Alternanthera philoxeroides)是苋 科莲子草属一种多年生草本植物, 适合在水生和陆 地生境中生长(Julien et al, 1995), 原产于阿根廷, 
1897年无意中被引入美国, 如今已广泛分布于包括 中国、美国和澳大利亚在内的许多国家和地区 (Burgin \& Norris, 2008)。自1985年以来, 它已成为 中国控制杂草的主要目标(Dong et al, 2015), 并于 2003年被中国环境保护总局列为中国首批外来入 侵物种。以往关于喜早莲子草引入地和原产地种群 的比较研究发现：喜旱莲子草引入地种群与耐阴性 有关的性状对光照的可塑性显著低于原产地种群 (张紫妍等, 2015); 引入地种群对专食性天敌的组 成抗性低于原产地种群(周方等, 2017); 引入地种 群与资源利用相关的功能性状(如根冠比和比叶面 积)对邻体根系的可塑性方向与原产地种群相反(于 良瑞等, 2020); 引入地种群亲属间的竞争反应高于 原产地种群(Zhang et al, 2019)等。关于喜旱莲子草 种群对增温表型可塑性的研究发现, 其引入地中国 的种群在全天增温 $0.76 \pm 0.10^{\circ} \mathrm{C}$ 条件下净光合速率 显著提高(+9.23\%) (王琼等，2017); 在全天增温 $1.4{ }^{\circ} \mathrm{C}$ 条件下生物量无显著变化(吴吴等, 2020); 在 全天增温 $2^{\circ} \mathrm{C}$ 条件下无性分株数无显著变化 $($ 褚延梅 等, 2014)。但是, 国内外学者尚未研究喜旱莲子草 引入地和原产地种群对增温响应的差异。

为了研究入侵植物引入地和原产地种群生物 量、重要功能性状和草食作用对增温响应是否存在 差异, 我们用喜早莲子草引入地(中国)和原产地(阿 根廷)的各 8 个种群，在广东省广州市增城区进行了 模拟全天增温 $2^{\circ} \mathrm{C}$ 的实验。测定的实验指标包括生 长指标、功能性状和草食损伤指标。本研究主要探 讨以下问题: (1)喜旱莲子草的生物量、功能性状和 被取食情况如何响应模拟全天增温? (2)这种响应在 引入地和原产地种群之间是否存在差异?

\section{1 材料与方法}

\section{1 实验地点}

模拟全天增温实验在广东省广州市增城区 $\left(113.87^{\circ} \mathrm{E}, 23.33^{\circ} \mathrm{N}\right)$ 进行。该地区属于北热带地区, 夏季炎热潮湿, 平均年气温 $21.8^{\circ} \mathrm{C}$, 年相对湿度 $80 \%$, 年平均降水量 $1,786 \mathrm{~mm}$ 。

\section{2 实验材料}

喜旱莲子草在原产地可以同时进行有性和无 性繁殖, 但是引入地种群的种子发育不充分且发芽 率极低, 扩繁的主要方法是通过储存根和茎节段进 行无性繁殖(潘晓云等, 2007)。本实验所用植物材料
是2018年从引入地(中国)和原产地(阿根廷)收集的 茎节获得, 每个来源地有 8 个种群, 基本上包括了 主要分布区域。每个种群至少收集20个茎段, 每个 茎段间距超过 $50 \mathrm{~m}$ 以避免重复收集同一克隆植株。 有关采样点信息参见附录 1 。所有收集的茎段均种 植在复旦大学江湾校区温室中, 培养三代以上以去 除母体效应。

实验基质为 $1: 1$ 的河沙和蛭石, 混匀后装入底 径 $7.5 \mathrm{~cm}$ 、口径 $12 \mathrm{~cm}$ 、高 $8 \mathrm{~cm}$ 的塑料花盆中。使用 MRM-2420 红外辐 射器 (Kalglo Electronics, Bethlehem, USA)进行加热, 该辐射器悬挂在土壤表 面上方 $2 \mathrm{~m}$ 处, 这种加热方式将目标植物周围的空 气温度提高了约 $2^{\circ} \mathrm{C}$ (在 $1.5-2.5^{\circ} \mathrm{C}$ 之间), 处于先前 研究预测的温度范围内(Peng et al, 2013)。在对照处 理组中, 使用一个与红外辐射器形状和尺寸相同的 “虚拟”加热器来模拟红外辐射器在未经加热的条件 下的遮光效果。

\section{3 实验设计}

2019年6月 10 日从每个种群切下 20 个大小相 似、长度约 $4 \mathrm{~cm}$ 且中间有一个茎节的茎段, 将其用 去离子水清洗3遍后放入塑料盒中培养。盒中铺 3 层 纸巾, 用去离子水保持湿润, 将茎段放进人工气候 培养箱(GXZ-380A，宁波)中培养萌发。培养条件设 置为白天 $28^{\circ} \mathrm{C}$ 、夜间 $25^{\circ} \mathrm{C}$ 、湿度 $60 \%$ 、光照 $12 \mathrm{~h}$ 。6 月22日将塑料盒及其中萌发的茎段由上海带至广 州实验地点，期间用去离子水保持茎段湿润。次日 将新发芽的茎段种植在盛有 $500 \mathrm{~mL}$ 混合基质的花 盆中, 每个花盆中种植一个茎段, 放在室内进行培 养。确认所有茎段均定植成功后，于6月25日从每个 种群中选择6株具有 2 对叶的生长状况良好且相似 的幼苗放入增温样地进行实验。两种处理分别为: 对照(环境温度)、模拟全天增温 $2^{\circ} \mathrm{C}$ 。每个处理设置 3 个重复, 共有 96 个实验单元 $(2$ 个来源地 $\times 8$ 个种群 $\times 2$ 种处理 $\times 3$ 个重复)。为保证生长过程植物所需养 分充足, 每周对所有植物施加 $50 \mathrm{~mL}$ 四倍不含氮的 标准霍格兰溶液和 $100 \mathrm{~mL}$ 的 $500 \mathrm{mg} / \mathrm{L}$ 氯化铵溶液。 其他条件包括辐照度、降水和草食性均在自然条件 下进行, 以模拟田间情况并观察气候变暖对两个来 源地种群的影响。实验采取完全随机排列，同时每 周将两种处理下的所有植物位置重新随机排列以 避免位置效应。每天观察植株状态，每周记录植物 的株高和分枝数, 实验进行 8 周, 所有植物于 2019 
年8月15日收获。

\section{4 指标测定}

测定指标为: (1)生物量, 包括总生物量(total biomass, TB)和聍藏根生物量(storage root biomass, $\mathrm{SRB}$ ); (2) 功能性状, 包括分枝强度 (branching intensity, BI)、比茎长(specific stem length, SSL)、根 冠比(root to shoot ratio, RSR)和比叶面积(specific leaf area, SLA); (3)食草作用, 包括相对取食面积 (relative feeding area, 被取食叶面积/总叶面积)和茎 端取食率(stem-tip feeding proportion, 被取食的茎 端数/茎端总数)。分枝强度为分枝生物量和主枝生 物量的比值, 较低的分枝强度能够减少植物地上部 分的种内竞争(Pan et al, 2013)。比茎长为植物主枝 长和主枝干重的比值, 即单位质量的主枝长度, 比 茎长越大, 表明植物茎秆越细。分枝强度和比茎长 反映了植物地上部分的形态(潘晓云等, 2007)。根冠 比为植物地下部和地上部干重的比值, 与植物对自 然资源的获取能力有关。当环境条件改变时, 植物 根冠比增大可以提高适合度和竞争能力(Shipley \& Meziane, 2002)。比叶面积即单位质量的叶面积, 与 植物对光资源的利用效率有关。

收获植物样品时, 用剪刀将植物从茎基部剪下, 记录所有茎端数量以及自然条件下被动物(棉大卷 叶螟(Sylepta derogata)、拟小黄卷叶蛾(Adoxophyes cyrtosema)等)取食的茎端数量; 然后将植物地上部 分的茎、叶分开, 用直尺测量主枝长度(精确到 0.1 $\mathrm{cm})$ 。植物地下部分是将花盆中基质倒出, 抖落植物 根系周围的土壤并清洗干净。将地上和地下部分分 别放入准备好的纸袋中, 取主枝上从上往下第三对 叶作为测量比叶面积的叶片。将第三对叶拍照留档, 用照片识别软件 Digimizer计算总叶面积(精确到 $0.01 \mathrm{~cm}^{2}$ ), 然后按照编号放入事先准备好的2寸纸 袋中。从所有叶片中随机选取4片作为测量草食作 用的叶片, 将其拍照留档, 用照片识别软件 Digimizer计算总叶面积和自然条件下被动物(东亚 飞蝗(Locusta migratoria manilensis)、虾钳菜披龟甲 (Cassida piperata)等)取食的叶面积(精确到 0.01 $\mathrm{cm}^{2}$ )。地上部分分离为第三对叶、其他叶片、主枝 和侧枝, 地下部分分离为贮藏根和细根。将分离出 的植物各部分置于 $60^{\circ} \mathrm{C}$ 烘箱中干燥至恒重, 再分别 使用电子分析天平 $(0.0001 \mathrm{~g})$ 称量, 得到各性状相关 数据。

\section{5 统计分析}

采用三因素方差分析检验各因素及其交互作 用对植物生物量、功能性状以及草食作用影响的显 著性, 所有检验均在 $P=0.05$ 的水平上进行 $(n=96)$ 。 采用R 3.6.0 (R Development Core Team, 2020)进行 分析并绘图。在R软件中调用nlme程序包, 采用线性 混合效应模型分析不同处理分别对各性状的影响。 在这些模型中, 将来源地(引入地vs. 原产地)、模拟 全天增温(对照vs.增温)及其相互作用作为固定效应, 种群作为随机效应嵌套于来源地内。用似然比检验 (likelihood-ratio tests)比较剔除固定因子对模型的影 响, 以评估混合效应模型里固定效应的显著性。在 进行方差分析之前，先用 Shapiro-Wilk normality tests检查数据的正态性和方差的均一性。对贮藏根 生物量和相对取食面积进行对数转换, 对分枝强 度、比叶面积和茎端取食率进行开平方转换, 以满 足残差的正态性。

\section{结果}

2.1 生物量、功能性状和草食作用对模拟全天增温 的响应及其在来源地间的差异

模拟全天增温显著降低了喜旱莲子草的总生物 量(-7.8\%)、败藏根生物量(-12.8\%)、分枝强度(-11.6\%) 和茎端取食率(-34.4\%) (表1; 图1a、b、c、h)。引入 地和原产地之间喜早莲子草的总生物量 $\left(\chi^{2}=2.986\right.$, $P=0.084)$ 、比茎长 $\left(\chi^{2}=3.453, P=0.063\right)$ 和相对取食 面积 $\left(\chi^{2}=3.682, P=0.055\right)$ 对模拟全天增温的可塑性 存在显著差异。和对照相比，模拟全天增温使原产地 种群总生物量下降 $2.1 \%$, 比茎长下降5.6\%, 相对取 食面积下降 $22.5 \%$, 而引入地种群总生物量下降 $13.0 \%$ ，比茎长增加 $13.2 \%$, 相对取食面积增加 $24.5 \%$ (表1; 图1a、d、g)。引入地和原产地种群间喜旱莲 子草的其他性状如聍藏根生物量、分枝强度、根冠 比、比叶面积和茎端取食率对模拟全天增温的可塑 性无显著差异(表1; 图1b、c、e、f、h)。

\section{2 引入地和原产地生物量、功能性状和草食作用} 的差异

与原产地种群相比, 喜旱莲子草引入地种群的 贮藏根生物量 $\left(+31.5 \%, \chi^{2}=8.756, P=0.003\right)$ 、分枝 强度 $\left(+38.5 \%, \chi^{2}=8.023, P=0.005\right)$ 、比茎长 $(+30.2 \%$, $\left.\chi^{2}=8.092, P=0.004\right) 、$ 根冠比 $\left(+24.5 \%, \chi^{2}=5.758, P\right.$ $=0.016)$ 和比叶面积 $\left(+20.0 \%, \chi^{2}=4.665, P=0.031\right)$ 



图1 喜旱莲子草引入地(中国)和原产地(阿根廷)种群对模拟全天增温的响应。误差棒代表 $95 \%$ 的置信区间; 表示全天增温处 理与来源地的交互作用弱显著。

Fig. 1 Responses of introduced (China) and native (Argentina) populations of Alternanthera philoxeroides to simulated daily warming. Error bars indicate 95\% confidence intervals, and $\dagger$ indicate marginally significant differences in responses between introduced and native populations.

表1 喜旱莲子草引入地(中国)和原产地(阿根廷)种群在两种处理(不增温、全天增温 $2^{\circ} \mathrm{C}$ )下生物量(总生物量、赔藏根生物量 和生长速率)、功能性状(分枝强度、比茎长、根冠比和比叶面积)和草食作用(相对取食面积和茎端取食率)的差异。字体加粗 表示效应显著 $(P<0.05)$, 加粗斜体表示弱显著 $(P<0.10)$ 。

Table 1 Effects of origin (i.e. introduced vs. native) and simulated daily warming (i.e. control vs. warming) and their interaction on fitness traits (total biomass, storage root biomass (STB)), functional traits (branching intensity (BI), specific stem length (SSL), root to shoot ratio (RSR) and specific leaf area (SLA)) and herbivorous effect (relative feeding area (RFA) and stem-tip feeding proportion (SFP)) of Alternanthera philoxeroides. Significant effects are marked in bold and marginally significant effects are marked in italics and bold.

\begin{tabular}{|c|c|c|c|c|c|c|c|c|c|c|c|c|c|c|c|c|}
\hline \multirow{2}{*}{$\begin{array}{l}\text { 变异来源 } \\
\text { Source of } \\
\text { variation }\end{array}$} & \multicolumn{2}{|c|}{$\begin{array}{l}\text { 总生物量 } \\
\text { Total biomass }\end{array}$} & \multicolumn{2}{|c|}{$\begin{array}{l}\text { 贠藏根生物量 } \\
\text { STB }\end{array}$} & \multicolumn{2}{|c|}{$\begin{array}{l}\text { 分枝强度 } \\
\mathrm{BI}\end{array}$} & \multicolumn{2}{|c|}{$\begin{array}{l}\text { 比茎长 } \\
\text { SSL }\end{array}$} & \multicolumn{2}{|c|}{$\begin{array}{l}\text { 根冠比 } \\
\text { RSR }\end{array}$} & \multicolumn{2}{|c|}{$\begin{array}{l}\text { 比叶面积 } \\
\text { SLAA }\end{array}$} & \multicolumn{2}{|c|}{$\begin{array}{l}\text { 相对取食面积 } \\
\text { RFA }\end{array}$} & \multicolumn{2}{|c|}{$\begin{array}{l}\text { 茎端取食率 } \\
\text { SFP }\end{array}$} \\
\hline & $\bar{\chi}^{2}$ & $P$ & $\chi^{2}$ & $P$ & $\chi^{2}$ & $P$ & $\chi^{2}$ & $P$ & $\chi^{2}$ & $P$ & $\chi^{2}$ & $P$ & $\chi^{2}$ & $P$ & $\chi^{2}$ & $P$ \\
\hline $\begin{array}{l}\text { 增温 } \\
\text { Warming }\end{array}$ & 5.165 & 0.023 & 3.207 & 0.073 & 4.239 & 0.040 & 0.761 & 0.383 & 0.343 & 0.558 & 0.021 & 0.884 & 0.083 & 0.773 & 10.552 & 0.001 \\
\hline $\begin{array}{l}\text { 来源地 } \\
\text { Origin }(\mathrm{O})\end{array}$ & 1.523 & 0.217 & 8.756 & 0.003 & 8.023 & 0.005 & 8.092 & 0.004 & 5.758 & 0.016 & 4.665 & 0.031 & 1.837 & 0.175 & 3.680 & 0.055 \\
\hline $\mathrm{W} \times \mathrm{O}$ & 2.986 & 0.084 & 0.244 & 0.621 & 1.077 & 0.299 & 3.453 & 0.063 & 0.913 & 0.339 & 0.365 & 0.546 & 3.682 & 0.055 & 1.772 & 0.183 \\
\hline
\end{tabular}


显著增加, 茎端取食率 $\left(-35.8 \%, \chi^{2}=3.680, P=\right.$ 0.055 )显著降低(表1; 图1b、c、d、e、f、h)。

\section{3 讨论}

\section{1 喜旱莲子草生物量、功能性状和草食作用对全} 天增温的响应

本研究发现, 喜旱莲子草在北热带地区(广州 市)模拟全天增温 $2^{\circ} \mathrm{C}$ 条件下总生物量、拒藏根生物 量显著降低。与此类似, 马铃薯(Solanum tuberosum) 的产量在全天增温 $4{ }^{\circ} \mathrm{C}$ 条件下显著降低 $(-30.3 \%)$ (Kim \& Lee, 2019)。增温导致生物量下降可能是由 于高温下植物光系统受损、光合作用受到限制 (Prange et al, 1990), 还可能由于增温导致气孔导度 降低、细胞衰老加快等原因(Prange et al, 1990)。但 也有与上述结果相反的研究报道, 如旱雀麦 (Bromus tectorum) 生物量和种子产量在增温 $\left(1.5{ }^{\circ} \mathrm{C} / 3{ }^{\circ} \mathrm{C}\right.$, 白天/夜晚)下增加 $200 \%$ 以上 (Blumenthal et al, 2016)。此外, 一项整合分析研究 也发现增温使陆地植物生物量显著增加 $(+12.3 \%)$ (Lin et al, 2010)。值得关注的是, 先前的研究发现喜 旱莲子草在温带全天增温 $1.4^{\circ} \mathrm{C}$ 条件下生物量无显 著变化(吴吴等, 2020), 这表明同一物种在不同气候 区的增温处理会出现不同的结果。

总体而言, 增温对植物生长促进或抑制的不同 结果可能有以下原因。首先, 增温实验不可避免地 涉及到不同植物物种和功能性状, 它们对增温反应 有所不同(Luo, 2007)。其次, 不同增温幅度和式样 (对称增温或不对称增温)的影响可能不同(Peng et $\mathrm{al}, 2013)$ 。第三，增温可以通过影响土壤水分和/或 土壤氮的可利用性来间接影响植物的生长(Lin et al, 2010)。因此, 在今后关于植物对增温响应的实验研 究中, 亟需全面考虑植物物种和功能类型、增温幅 度和式样、以及增温改变土壤湿度和养分可利用性 的间接效应等因素的综合影响。

除了对生长的影响, 我们的研究还发现, 模拟 全天增温条件下喜旱莲子草分枝强度和茎端取食 率降低, 表明增温可能提高植物的抗性或耐受性。 与此结果相似, 番茄(Solanum lycopersicum)在全天 增温 $4.5^{\circ} \mathrm{C}$ 条件下叶片防御物质胰蛋白酶抑制剂和 叶毛密度均显著提高(Paudel et al, 2020)。花椰菜 (Brassica oleracea) 芥子油式含量在增温条件下(对 照组: $18^{\circ} \mathrm{C} / 12^{\circ} \mathrm{C}, 22^{\circ} \mathrm{C} / 15^{\circ} \mathrm{C}$; 实验组: $30^{\circ} \mathrm{C} / 15^{\circ} \mathrm{C}$;
白天/夜晚)显著提高(Pereira et al, 2002)。也有一些 研究与我们的结果不同, 如飞廉(Carduus nutans)的 叶刺密度在增温条件(对照组: 环境温度; 实验组: 增温0.58 ${ }^{\circ} \mathrm{C}$ )下显著降低(Zhang et al, 2012)。这些结 果表明, 不同植物的抗性和耐受性对增温的响应可 能不同(Lemoine et al, 2013)。因此, 研究植物防御性 状对增温的响应应考虑植物防御策略(如诱导抗性 或耐受性)和草食动物类型(如专化昆虫或泛化昆虫) 的综合影响(Paudel et al, 2020)。

\section{2 喜旱莲子草生物量、功能性状和草食作用对全} 天增温的响应在引入地和原产地间的差异

本研究发现, 喜旱莲子草生物量、比茎长和相 对取食面积对模拟全天增温的响应在引入地和原 产地间存在差异 $(0.05<P<0.1)$ 。引入地种群在模拟 全天增温处理下的生物量下降比例是原产地种群 的7倍, 这表明引入地种群生物量对增温的响应更 强, 且符合 “master-of-some”的范式(Richards et al, 2006), 即在更有利的环境下适合度提高。在我们的 研究中, 热带不增温条件对于喜早莲子草是更有利 的环境, 而全天增温 $2^{\circ} \mathrm{C}$ 对其则是胁迫的环境。比茎 长是植物对资源利用效率的一个重要指标, 比茎长 越大, 植物的茎秆越细长, 植物运输养分的效率越 低(Pan et al, 2013)。本研究结果表明, 引入地种群的 比茎长对模拟全天增温的可塑性大于原产地种群, 这一结果支持表型可塑性增强进化假说(Richards et al, 2006)。

目前关于外来植物适合度和功能性状对增温 的响应在引入地和原产地之间是否存在差异, 以及 如果存在差异是否符合表型可塑性进化增强假说 存在很大争议。首先, 有研究发现引入地和原产地 植物旱雀麦的生长相关性状(生物量、资源分配、叶 长和光合作用)对增温 (对照组: $10^{\circ} \mathrm{C} / 5^{\circ} \mathrm{C}$; 实验组: $30{ }^{\circ} \mathrm{C} / 20^{\circ} \mathrm{C}$; 白天/夜晚) 的可塑性没有显著差异 (Griffith et al, 2014)。其次, 也有研究发现, 引入地 和原产地种群生长的相关性状对增温的响应方向 不同。如喜盐草(Halophila stipulacea)引入地种群茎 数量和生长速率对增温 (对照组: $26^{\circ} \mathrm{C}$; 实验组: $29^{\circ} \mathrm{C} 、 32^{\circ} \mathrm{C}$ )无响应, 叶形大小对增温有正响应, 而 原产地种群茎数量对增温表现为负响应(Nguyen et al, 2020)。加拿大一枝黄花(Solidago canadensis)引 入地种群的种子发芽时间对增温(对照组: 环境温 度; 实验组: 增温 $2^{\circ} \mathrm{C}$ )无响应, 而原产地种群在增 
温条件下种子发芽时间显著提前; 引入地种群种子 发芽率在增温条件下显著降低, 原产地种群则显著 增加(Zhou \& He, 2020)。第三, 引入地和原产地种 群的生物量和生长相关性状在增温条件下显著增 加(这与本研究结果相反)。在此情形下, 引入地种群 响应大于原产地种群的例子有：金鱼藻 (Ceratophyllum demersum)引入地种群相对生长速 率和光合作用对增温 $\left(\right.$ 对照组 $12^{\circ} \mathrm{C}$; 实验组: $18^{\circ} \mathrm{C}$ 、 $25^{\circ} \mathrm{C} 、 35^{\circ} \mathrm{C}$ )的可塑性大于原产地种群(Hyldgaard \& Brix, 2012); 加拿大一枝黄花引入地种群叶干物质 量对增温的可塑性大于原产地种群(Zhou \& He, 2020); 西洋萻草(Achillea millefolium)引入地种群种 子发芽率对增温(对照组: $8{ }^{\circ} \mathrm{C} / 4^{\circ} \mathrm{C}$, 白天/夜晚; 实 验组: $20^{\circ} \mathrm{C} / 10^{\circ} \mathrm{C}, 32^{\circ} \mathrm{C} / 20^{\circ} \mathrm{C}$, 白天/夜晚)的可塑性 大于原产地种群(Beckmann et al, 2011); 绿毛山柳 菊(Hieracium pilosella)引入地种群种子发芽率对增 温(对照组: $8^{\circ} \mathrm{C} / 4^{\circ} \mathrm{C}$; 实验组: $20^{\circ} \mathrm{C} / 10^{\circ} \mathrm{C}$; 白天/夜晚) 的可塑性大于原产地种群(Beckmann et al, 2011)。相 反, 引入地种群的响应小于原产地种群的案例也有, 如加拿大一枝黄花引入地种群分枝数和开花时间 对增温(对照组: 环境温度; 实验组: 增温 $2^{\circ} \mathrm{C}$ )的可 塑性小于原产地种群(Peng et al, 2019)。最后, 引入 地和原产地种群的生物量和生长相关性状对增温 响应均表现为减小且引入地种群的响应小于原产 地种群。如斑点矢车菊(Centaurea maculosa)引入地 种群的生物量对增温(对照组: 环境温度; 实验组: 增温 $1.5-2.5^{\circ} \mathrm{C}$ ) 的响应小于原产地种群 (He et al, 2012)。

因此，目前关于外来植物适合度和功能性状对 增温的响应在引入地和原产地之间是否存在差异, 以及如果存在差异是否符合表型可塑性增强进化 假说并没有一致的结论, 需要进行更深入的研究。

和对照相比，模拟全天增温条件下植物的相对 取食面积在引入地(+24.5\%)和原产地(-22.5\%)表现 出相反的趋势, 表明在模拟全天增温条件下, 引入 地种群对草食作用的抗性降低, 而原产地种群的抗 性增加。这种可塑性反应的绝对值相似而方向相反 的式样并未被表型可塑性增强进化假说(主要关注 可塑性方向一致而绝对值大小存在差异)所描述(于 良瑞等, 2020)。据我们所知, 关于增温条件下入侵 植物草食作用在引入地和原产地之间差异的研究 尚未见报道。
上述结果表明, 与原产地种群相比, 热带地区 全天增温使喜早莲子草引入地种群生物量显著下 降、草食作用显著增加, 表明在未来全球气候变暖 的背景下, 热带地区温度升高可能不利于其种群多 度的增加。本研究结果表明, 喜旱莲子草引入地和 原产地种群在生物量和重要功能性状出现显著分 化。如引入地种群的聍藏根生物量显著高于原产地 种群, 所有功能性状均高于原产地种群, 茎端取食 率低于原产地种群。以上结果与我们之前的同质园 实验结果既有相同也有不同之处。例如，有两项研 究发现引入地种群的总生物量显著高于原产地种 群(周方等, 2017; 于良瑞等, 2020), 一项研究发现 引入地种群总生物量低于原产地种群(Portela et al. 2020), 但也有两项研究中生物量无差异(张紫妍等, 2015; Liu et al, 2018)。此外, 有研究发现喜早莲子 草引入地种群对泛化天敌的组成抗性高于原产地 种群，但对专化天敌的耐受性以及对泛化天敌的诱 导性防御低于原产地种群(Liu et al, 2020)。上述研 究中引入地种群的相关功能性状均低于原产地。这 种部分性状的不一致性可能是由于实验材料和实 验条件差异等造成, 如本研究使用的喜早莲子草引 入地种群来自中国, 而以上研究中喜早莲子草的引 入地种群均来自美国。而防御性状结果的差异可能 与取食昆虫的种类以及防御物质种类不同有关。同 时各研究中施加的处理均有差异，也可能对植物造 成了不同的影响。此外，实验使用的基质、进行时 间和开展地点均可能对植物性状造成不同影响。

\section{ORCID}

潘晓云 (D) https://orcid.org/0000-0001-8421-8960 吴纪华 (iD https://orcid.org/0000-0001-8623-8519

\section{参考文献}

Beckmann M, Bruelheide H, Erfmeier A (2011) Germination responses of three grassland species differ between native and invasive origins. Ecological Research, 26, 763-771.

Blumenthal DM, Kray JA, Ortmans W, Ziska LH, Pendall E (2016) Cheatgrass is favored by warming but not $\mathrm{CO}_{2}$ enrichment in a semi-arid grassland. Global Change Biology, 22, 3026-3038.

Buckland SM, Thompson K, Hodgson JG, Grime JP (2001) Grassland invasions: Effects of manipulations of climate and management. Journal of Applied Ecology, 38, 301-309.

Burgin S, Norris A (2008) Alligator weed (Alternanthera philoxeroides) in New South Wales, Australia: A status 
report. Weed Biology and Management, 8, 284-290.

Chu YM, Yang J, Li JJ, Peng PH (2014) Three warming scenarios differentially affect the morphological plasticity of an invasive herb Alternanthera philoxeroides. Acta Ecologica Sinica, 34, 1411-1417. (in Chinese with English abstract) [褚延梅, 杨健, 李景吉, 彭培好 (2014) 三种增 温情景对入侵植物空心莲子草形态可塑性的影响. 生态 学报, 34, 1411-1417.]

Daehler CC (2003) Performance comparisons of co-occurring native and alien invasive plants: Implications for conservation and restoration. Annual Review of Ecology, Evolution, and Systematics, 34, 183-211.

Dong BC, Alpert P, Zhang Q, Yu FH (2015) Clonal integration in homogeneous environments increases performance of Alternanthera philoxeroides. Oecologia, 179, 393-403.

Geng YP, Pan XY, Xu CY, Zhang WJ, Li B, Chen JK (2006) Phenotypic plasticity of invasive Alternanthera philoxeroides in relation to different water availability, compared to its native congener. Acta Oecologica, 30, 380-385.

Griffith AB, Andonian K, Weiss CP, Loik ME (2014) Variation in phenotypic plasticity for native and invasive populations of Bromus tectorum. Biological Invasions, 16, 2627-2638.

He WM, Li JJ, Peng PH (2012) Simulated warming differentially affects the growth and competitive ability of Centaurea maculosa populations from home and introduced ranges. PLoS ONE, 7, e31170.

Hyldgaard B, Brix H (2012) Intraspecies differences in phenotypic plasticity: Invasive versus non-invasive populations of Ceratophyllum demersum. Aquatic Botany, 97, 49-56.

Julien MH, Skarratt B, Maywald GF (1995) Potential geographical-distribution of alligator weed and its biological-control Agasicles hygrophila. Journal of Aquatic Plant Management, 33, 55-60.

Kim YU, Lee BW (2019) Differential mechanisms of potato yield loss induced by high day and night temperatures during tuber initiation and bulking: Photosynthesis and tuber growth. Frontiers in Plant Science, 10, 300.

Lemoine NP, Drews WA, Burkepile DE, Parker JD (2013) Increased temperature alters feeding behavior of a generalist herbivore. Oikos, 122, 1669-1678.

Lin DL, Xia JY, Wan SQ (2010) Climate warming and biomass accumulation of terrestrial plants: A meta-analysis. New Phytologist, 188, 187-198.

Liu M, Pan XY, Zhang ZJ, van Kleunen M, Li B (2020) Testing the shifting defense hypothesis for constitutive and induced resistance and tolerance. Journal of Pest Science, 93, 355-364.

Liu M, Zhou F, Pan XY, Zhang ZJ, Traw MB, Li B (2018) Specificity of herbivore-induced responses in an invasive species, Alternanthera philoxeroides (Alligator weed).
Ecology and Evolution, 8, 59-70.

Luo YQ (2007) Terrestrial carbon-cycle feedback to climate warming. Annual Review of Ecology Evolution and Systematics, 38, 683-712.

McKinney ML (2006) Urbanization as a major cause of biotic homogenization. Biological Conservation, 127, 247-260.

Nguyen HM, Yadav NS, Barak S, Lima FP, Sapir Y, Winters G (2020) Responses of invasive and native populations of the seagrass Halophila stipulacea to simulated climate change. Frontiers in Marine Science, 6, 812.

Nicotra AB, Atkin OK, Bonser SP, Davidson AM, Finnegan EJ, Mathesius U, Poot P, Purugganan MD, Richards CL, Valladares F, van Kleunen M (2010) Plant phenotypic plasticity in a changing climate. Trends in Plant Science, 15, 684-692.

Pan XY, Geng YP, Sosa A, Zhang WJ, Li B, Chen JK (2007) Invasive Alternanthera philoxeroides: Biology, ecology and management. Acta Phytotaxonomica Sinica, 45, 884-900. (in Chinese with English abstract) [潘晓云, 耿宇鹏, Alejandro Sosa, 张文驹, 李博, 陈家宽 (2007) 入侵植物 喜旱莲子草—生物学、生态学及管理. 植物分类学报, 45, 884-900.]

Pan XY, Jia X, Fu DJ, Li B (2013) Geographical diversification of growth-defense strategies in an invasive plant. Journal of Systematics and Evolution, 51, 308-317.

Paudel S, Lin PA, Hoover K, Felton GW, Rajotte EG (2020) Asymmetric responses to climate change: Temperature differentially alters herbivore salivary elicitor and host plant responses to herbivory. Journal of Chemical Ecology, 46, 891-905.

Peng SS, Piao SL, Ciais P, Myneni RB, Chen AP, Chevallier F, Dolman AJ, Janssens IA, Peñuelas J, Zhang GX, Vicca S, Wan SQ, Wang SP, Zeng H (2013) Asymmetric effects of daytime and night-time warming on Northern Hemisphere vegetation. Nature, 501, 88-92.

Peng Y, Yang JX, Zhou XH, Peng PH, Li JJ, Zhang SM, He WM (2019) An invasive population of Solidago canadensis is less sensitive to warming and nitrogen-addition than its native population in an invaded range. Biological Invasions, 21, 151-162.

Pereira FMV, Rosa E, Fahey JW, Stephenson KK, Carvalho R, Aires A (2002) Influence of temperature and ontogeny on the levels of glucosinolates in broccoli (Brassica oleracea var. italica) sprouts and their effect on the induction of mammalian phase 2 enzymes. Journal of Agricultural and Food Chemistry, 50, 6239-6244.

Portela R, Dong BC, Yu FH, Barreiro R, Roiloa SR, Silva Matos DM (2020) Trans-generational effects in the clonal invader Alternanthera philoxeroides. Journal of Plant Ecology, 13, 122-129.

Prange RK, McRae KB, Midmore DJ, Deng RB (1990) Reduction in potato growth at high temperature: Role of photosynthesis and dark respiration. American Potato 
Journal, 67, 357-369.

R Development Core Team (2020) R: A Language and Environment for Statistical Computing. R Foundation for Statistical Computing, Vienna.

Richards CL, Bossdorf O, Muth NZ, Gurevitch J, Pigliucci M (2006) Jack of all trades, master of some? On the role of phenotypic plasticity in plant invasions. Ecology Letters, 9, 981-993.

Shipley B, Meziane D (2002) The balanced-growth hypothesis and the allometry of leaf and root biomass allocation. Functional Ecology, 16, 326-331.

Wang Q, Tang Y, Xie T, Wang H (2017) Distinctive responses of photosynthetic characteristics to warming of invasive Alternanthera philoxeroides and native Sambucus chinensis. Acta Ecologica Sinica, 37, 770-777. (in Chinese with English abstract) [王琼, 唐娅, 谢涛, 王辉 (2017) 入侵植 物喜旱莲子草和本地种接骨草光合生理特征对增温响应 的差异. 生态学报, 37, 770-777.]

Wu H, Zhang C, Dai WK (2020) Interactive effects of climate warming and species diversity on the invasiveness of the alien weed Alternanthera philoxeroides. Acta Prataculturae Sinica, 29, 38-48. (in Chinese with English abstract) [吴吴, 张辰, 代文鬼 (2020) 气候变暖和物种多样性交互效应 对空心莲子草入侵的影响. 草业学报, 29, 38-48.]

Yu LR, Zhu ZC, Pan XY (2020) Phenotypic plasticity of Alternanthera philoxeroides in response to root neighbors of kin: Introduced vs. native genotypes. Biodiversity Science, 28, 651-657. (in Chinese with English abstract) [于良瑞, 朱政财, 潘晓云 (2020) 喜旱莲子草对同基因型邻体根
系的表型可塑性: 入侵地和原产地的比较. 生物多样性, 28, 651-657.]

Zhang R, Leshak A, Shea K (2012) Decreased structural defence of an invasive thistle under warming. Plant Biology, 14, 249-252.

Zhang ZJ, Zhou F, Pan XY, Kleunen M, Liu M, Li B (2019) Evolution of increased intraspecific competitive ability following introduction: The importance of relatedness among genotypes. Journal of Ecology, 107, 387-395.

Zhang ZY, Zhang ZJ, Pan XY (2015) Phenotypic plasticity of Alternanthera philoxeroides in response to shading: Introduced vs. native populations. Biodiversity Science, 23, 18-22. (in Chinese with English abstract) [张紫妍, 张致杰, 潘晓云 (2015) 喜旱莲子草对遮荫的可塑性反应: 入侵 地与原产地种群的比较. 生物多样性, 23, 18-22.]

Zhou F, Zhang ZJ, Liu M, Pan XY (2017) Effects of nutrient levels on defense against specialist insects in an invasive alligator weed. Biodiversity Science, 25, 1276-1284. (in Chinese with English abstract) [周方, 张致杰, 刘木, 潘晓 云 (2017) 养分影响入侵种喜旱莲子草对专食性天敌的 防御. 生物多样性, 25, 1276-1284.]

Zhou XH, He WM (2020) Climate warming facilitates seed germination in native but not invasive solidago canadensis populations. Frontiers in Ecology and Evolution, 8, 595214.

\section{附录 Supplementary Material}

附录1 喜旱莲子草原产地(阿根廷)和引入地(中国)种群采样点

Appendix 1 Sampling sites of Alternanthera philoxeroides from native (Argentina) introduced (China) range http://www.biodiversity-science.net/fileup/PDF/2020387-1.pdf 
黄河燕, 朱政财, 吴纪华, 拉琼, 周永洪, 潘晓云 (2021) 喜早莲子草对模拟全天增温的可塑性: 引入地和原产地种群的比较. 生物多样性, 29 (4): $419-$ 427. http://www.biodiversity-science.net/CN/10.17520/biods.2020387

附录1 喜旱莲子草原产地(阿根廷)和引入地(中国)种群采样点

Appendix 1 Sampling sites of Alternanthera philoxeroides from native (Argentina) introduced (China) range

\begin{tabular}{lllll}
\hline 采样点 Sampling site & 纬度 Latitude & 经度 Longitude & 生境 Habitat & 年均温 Annul mean temperature $\left({ }^{\circ} \mathrm{C}\right)$ \\
\hline 阿根廷(原产地) & $25^{\circ} 8^{\prime} \mathrm{S}$ & $58^{\circ} 14^{\prime} \mathrm{W}$ & 22.66 \\
Argentina (Native) & $27^{\circ} 25^{\prime} \mathrm{S}$ & $58^{\circ} 50^{\prime} \mathrm{W}$ & 路边 & 21.07 \\
& $28^{\circ} 28^{\prime} \mathrm{S}$ & $59^{\circ} 0^{\prime} \mathrm{W}$ & 路边 & 20.61 \\
& $30^{\circ} 2^{\prime} \mathrm{S}$ & $59^{\circ} 31^{\prime} \mathrm{W}$ & 路边 & 19.64 \\
& $31^{\circ} 33^{\prime} \mathrm{S}$ & $59^{\circ} 12^{\prime} \mathrm{W}$ & 路边 & 18.42 \\
& $33^{\circ} 27^{\prime} \mathrm{S}$ & $58^{\circ} 48^{\prime} \mathrm{W}$ & 路边 & 17.63 \\
& $35^{\circ} 56^{\prime} \mathrm{S}$ & $58^{\circ} 59^{\prime} \mathrm{W}$ & 路边 & 15.61 \\
& $37^{\circ} 49^{\prime} \mathrm{S}$ & $58^{\circ} 13^{\prime} \mathrm{W}$ & 路边 & 13.89 \\
\hline 中国(引入地) & $22^{\circ} 58^{\prime} \mathrm{N}$ & $113^{\circ} 13^{\prime} \mathrm{E}$ & 路边 & 22.25 \\
China (Introduced) & $24^{\circ} 0^{\prime} \mathrm{N}$ & $114^{\circ} 48^{\prime} \mathrm{E}$ & 池塘边 & 21.26 \\
& $25^{\circ} 54^{\prime} \mathrm{N}$ & $115^{\circ} 2^{\prime} \mathrm{E}$ & 农田 & 19.19 \\
& $28^{\circ} 33^{\prime} \mathrm{N}$ & $115^{\circ} 56^{\prime} \mathrm{E}$ & 农田 & 17.90 \\
& $30^{\circ} 42^{\prime} \mathrm{N}$ & $116^{\circ} 49^{\prime} \mathrm{E}$ & 农田 & 16.73 \\
& $32^{\circ} 39^{\prime} \mathrm{N}$ & $116^{\circ} 37^{\prime} \mathrm{E}$ & 农田 & 15.58 \\
& $34^{\circ} 22^{\prime} \mathrm{N}$ & $117^{\circ} 14^{\prime} \mathrm{E}$ & 路边 & 14.73 \\
& $35^{\circ} 26^{\prime} \mathrm{N}$ & $116^{\circ} 38^{\prime} \mathrm{E}$ & 河边 & 14.15 \\
\hline
\end{tabular}

